\title{
Tactical Robot Prototype for SWAT and Emergency Response Teams in Peru
}

\author{
Jason Chicoma-Moreno $^{1}$, and Junior Yllatopa-Turpo ${ }^{1}$ \\ 1Universidad de Ciencias y Humanidades, Perú, jasonchicoma@ieee.org,junyllatopat@uch.pe
}

\begin{abstract}
The lack of technology to explore hostile areas in Peru is an important factor for the increment of casualties among the staff of the Peruvian police and military. In view of this, we propose the design and construction of a tactical robot for the exploration of hostile zones in Peru which will be controlled with a telemetry system. For the first prototype, the most important parameters considered are the control of the unmanned vehicle, the transmission of video in real time and the fact that it is an all-terrain vehicle. To maintain the control of the tactical robot with the parameters mentioned above, a software was developed in Arduino Uno which is based on the $C++$ programming language. With this software the tactical robot can be controlled and monitored through a wireless network by accessing it through an IP address. We have to work further on the wireless communication, increasing security protocols, which will be implemented in future implementations of this project. It is expected that this tactical robot will be used by the police or military forces, so that they can make reconnaissance of hostile areas and thus their staff will be more prepared at the time of interventions.
\end{abstract}

Keywords-- Arduino, tactical robot, telemetry, Wi-Fi, smartphone

\section{INTRODUCTION}

A robot can perform several types of work depending on what their main purpose is. Robots are designed to perform tasks that people may or may not be able to do by themselves. Mobile robots and autonomous vehicles have experienced a great evolution in recent years [1]. Nowadays, we use these robots and machines all over the world. The use of robots as autonomous vehicles on the battlefield and in industry is unquestionable. Numerous and complex research studies are working on the development of autonomous air, land and underwater robots [2].

The unmanned ground vehicles (UGV) robotic system is an integration of several technologies including both hardware and software. Careful planning and execution are required to achieve the aforementioned undertaking [3]. Before choosing the right type of microcontroller for the robot control system, it is important to first identify the purpose of this [4].

For all the above there is a need for ground robots that can move around in the hills and muddy areas. Using ClosedCircuit Cameras several technical advances are made for surveillance as well [5].

The Micro Tactical Ground Robot (MTGR) manufactured by ROBOTEAM, was designed and developed for difficult field conditions. It includes a platform with a 4-DOF manipulator and a robust operator control unit 7 (ROCU-7). The manipulator can exert a maximum grip force of $186 \mathrm{~N}$. The MTGR Explosive Ordnance Disposal (EOD) system is

Digital Object Identifier (DOI):

http://dx.doi.org/10.18687/LACCEI2020.1.1.89

ISBN: 978-958-52071-4-1 ISSN: 2414-6390 currently used by special forces and tactical and urban warfare units [6].

Cameleon LG E developed by ECA GROUP developed a lightweight, high strength mini UGV dedicated to Electronic Data Interchange (EDI) and Explosive Ordnance Disposal (EOD) missions. Its modular architecture and Plug \& Play systems allow a wide range of payloads and sensors to be mounted, from manipulator arms to detection and neutralization sensors and water disrupters. The CAMELEON LG is designed to deploy quickly in the field without slowing down or hindering tactical movements. This robot can be carried in a backpack in addition to the standard equipment that an infantryman carries when deployed on OPEX (Overseas Operations) missions [7].

The LT2 designed by SDR TACTICAL ROBOTS is capable of climbing ladders and domestic obstacles. For more aggressive terrain and steeper stairs, the LT2-F includes rear stabilizer/flipper arms, which are used to raise the robot's nose over high obstacles. They also act as stabilizers to prevent tipping over on steep slopes [8].

EOD Mini-CALIBER developed by ICOR TECHNOLOGY has a Command and Control Unit (CCU) with multi-function control and variable speed joystick. In addition to a Radio Frequency (RF) wireless data and video system with two-way communication (talk/listen) with omnidirectional microphone and PTT (Push To Talk) functionality, this robot has a motor system two-way communication (speaking/listening) with omnidirectional microphone and PTT (Push To Talk) functionality and front and rear articulation wings for climbing stairs and clearing obstacles [9].

In the development of the UGV, the NATIONAL TATICAL OFFICERS ASSOCIATION (NTOA) seeks to establish a partnership to streamline the exchange of information between U.S. police tactical teams [10].

In Peru, the tactical implementation for reconnaissance of urban hostile areas is practically null, making it a danger for the staff of the police and military when exploring such areas. The present work, therefore, tries to reduce that risk towards the forces of the law and order having an implementation of a tactical mobile robot with a system of telemetry that will serve for exploration of hostile zones before intervention. In order to achieve this objective, we present the design and construction of a tactical robot for urban areas in Peru. In section II, the Methodology will indicate the mechanical and electronic parts needed for the project and the software developed for the control of the tactical robot. Section III presents the mechanical and electronic design and the results generated according to the tests that were carried out. Finally, in section IV the conclusions and future improvements of the implementation of the tactical robot will be indicated. 


\section{METHODOLOGY}

\section{A. Electronic Parts}

Since it is a prototype towards a tactical robot, simulation tests had to be done in order to choose the following electronic components for the optimal functioning of the robot:

- Arduino uno

- L293D Driver

- Module AR9331

- ESP Cam 32

- Lithium matter $12 \mathrm{v} 4000 \mathrm{mAh}$

The camera ESP Cam 32 is used to be able to have a visualization of a real time environment by means of the AR9331 module, which is a wifi real time video transmission module. This module will send the video image in real time to the mobile device that is connected wirelessly. Using the Arduino One with the help of the L293D driver, it will be possible to control the gearmotors for the movement of the tactical robot. The use of the wireless communication will be through the AR9331 module to connect it to the mobile device that will be able to control the tactical robot. Likewise, the tactical robot will be controlled through an interface developed in appInventor. This application will make possible to visualize and control the tactical robot. Besides, this interface created in appInventor will be in charge of sending the specific commands for the displacement and the actions that the tactical robot will perform. The whole interconnection between electronic components can be seen in Fig. 1.



Fig. 1 Tactical Robot Electronic Diagram

\section{B. Mechanical Parts}

The tactical robot must be robust, since it has to be an allterrain vehicle. For this prototype, the following resistant mechanical materials will be used to comply with the type of displacement that is expected:

- PVC Chassis

- Geared motor

- Tires

- Servo Motor

- Gimbal System

- Screw assembly

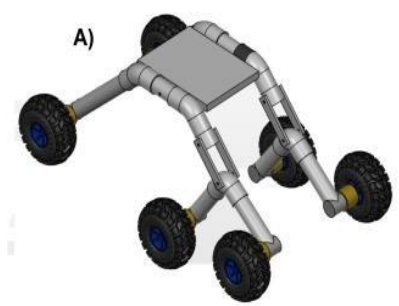

B)
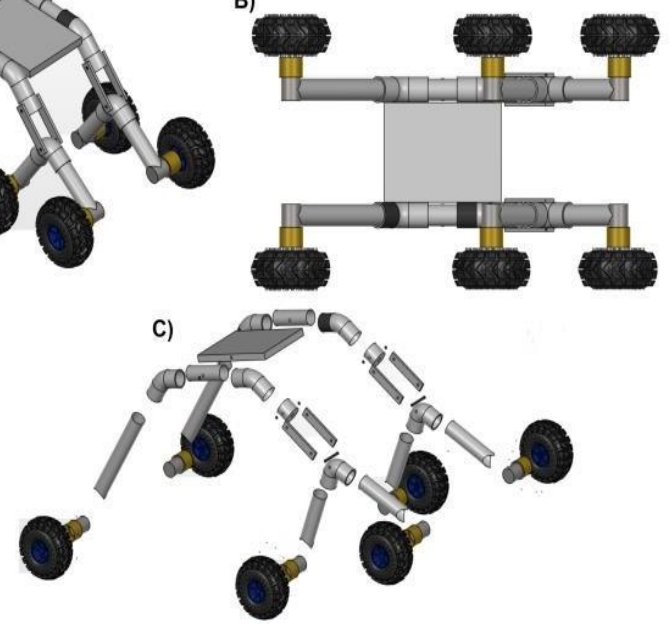

Fig. 2 3D mechanical diagram of the Tactical Robot. A) Isometric View. B) Bottom View. C) Fragmented View

Fig. 2 shows the mechanical construction of the tactical robot. In this prototype the chassis is made of acrylic to test its strength, durability and weight. The gearmotors will have a great performance at the moment of the displacement since it will be necessary a required force to be able to pass diverse type of obstacles. Thanks to their design inspired by a robot bogie, our tactical robot is ideal for an all-terrain vehicle. For this purpose, it is also effective to have a model of standardized tires for an all-terrain vehicle, since the type of waterproofing that the tire has will be vital for the performance of the tactical robot's movement. To ensure this, we developed a diagram (Fig. 2) using a 3D modulation application called Grabcad, that thanks to its user-friendly design interface it was possible to elaborate the design required.

\section{Software}

Since the tactical robot will be manipulated at long distance, an interface was developed using appInventor. This is an Android application creation system developed by the Michigan Institute of Technology (MIT). This software allows to program in blocks. The interface is used to send commands to the Arduino uno for the control of the tactical robot. Once the command is sent to the Arduino uno through the interface developed for the Android mobile device, it analyzes it and executes the action set by that command. To do this, the code in Arduino is partitioned into 3 parts.

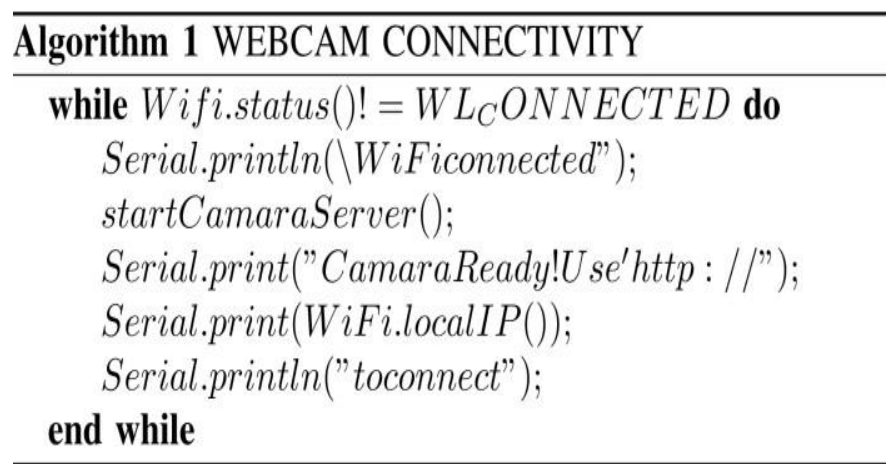

$18^{\text {th }}$ LACCEI International Multi-Conference for Engineering, Education, and Technology: "Engineering, Integration, and Alliances for a Sustainable Development" "Hemispheric Cooperation for Competitiveness and Prosperity on a Knowledge-Based Economy", 29-31 July 2020, Buenos Aires, Argentina. 
The first part consists of the webcam connectivity. As shown in algorithm 1 , it is used for initialization and continuity of the Wi-Fi connection. This allows the visualization of the environment through the webcam in real time by using the interface developed in the mobile device.

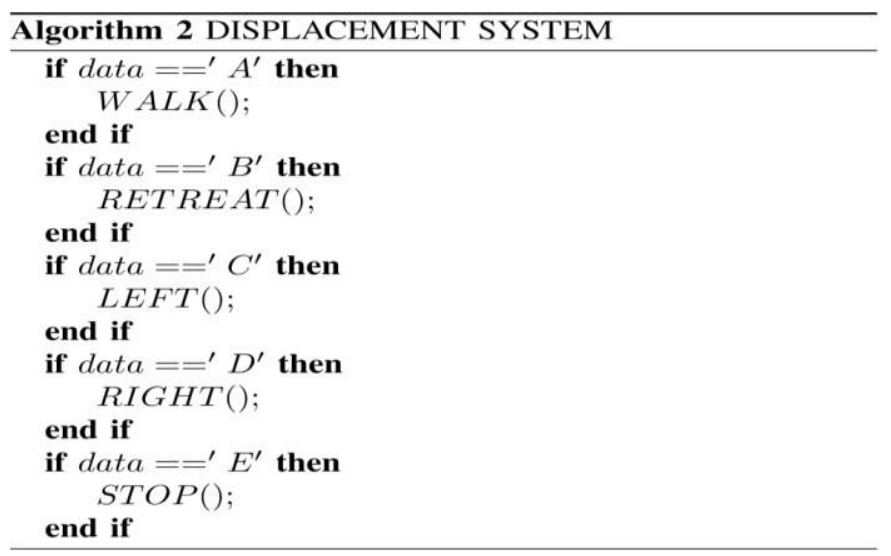

The second part is the displacement system. The purpose of this part can be seen in the algorithm 2 which is for the displacement of the tactical robot. Through the Android interface, the commands are sent, and the Arduino analyzes them and executes the action established for each case.

\begin{tabular}{l}
\hline Algorithm 3 DISPERSION SYSTEM \\
\hline if $d a t a==^{\prime} F^{\prime}$ then \\
$\quad S M O K E_{P} U M P() ;$ \\
end if
\end{tabular}

The dispersion system shown in algorithm 3 is used for the dispersion drive against hostile forces. For this purpose, this system is a built-in non-lethal smoke bomb. This part of the mechanism will be very useful for a law enforcement intervention against hostile threats.

\section{TESTES UNDERTAKEN}

The beginning of the tests of the following paper, was carried out in Lima - Peru. Due to the fact that in the different capitals of Peru, they have different geographical and environmental situations, where these factors derive much in the wireless communication that this first prototype has. Access to wireless networks in rural areas of Peru is usually very limited, this prototype would not be functional in those rural areas in its current state.

The environmental conditions and access to wireless networks for this first prototype were optimal, for its higher performance, as indicated above. The initial tests took place in Lima - Peru. To be more precise, in the district of Los Olivos, where a circuit was mounted, testing the torque of the motors in the ascent of inclined steps with the following angles: $15^{\circ}$, $35^{\circ}, 45^{\circ}$ and $75^{\circ}$. In these steps they were flat, where it could be noticed the efficiency of the motors, which had no complications in the $15^{\circ}, 35^{\circ}$ and $45^{\circ}$ steps. In the $75^{\circ}$ flat step they had complications where the prototype presented instability.
Taking into account the angle of instability that the prototype presented, test were made to climb steps to measure the time it takes to climb each step. This test was done with a stopwatch which averaged 1.23 seconds per step. Which in proportion to be the first prototype has a good upload time, but in the same way this time can be improved with another type of system. It is being considered for the next prototype to to build it in the shape of a caterpillar divided into 3 parts so that it can have more stability and speed in climbing steps.

Apart from the step tests, tests were made in which he was presented with a more rural terrain where he passed through branches, stones and pools of water. The biggest problem, the first prototype had, was to go through the type of terrain that was completely covered branches, due to its current structure. Once again favoring the idea of having the second version in a caterpillar type design. In the parts where there were stones and puddles of water, the prototype had no problem, but it was possible to notice that when the puddles were passed, the engine had to be covered so that it would not be damaged by the water. Because of that, another point is taken to improve the design that could be counting in the future the second structure of the prototype.

For ease of wireless communication in this first prototype was designed the operation of this, taking into account the Wifi network being the most susceptible. But if it is taken to rural areas where access to this type of network is very limited, they would not have the same range of response in real time, which means it would be inefficient. For testing in this first version using the Wifi network or the wireless data access you may be counting. The following test was made: First with access to mobile data it was taken to open field where I can notice that this had fluidity of movement and video transmission in real time, where it presented complication in only the part of video transmission were to the 15 meters approximate of the mobile device with which it was being controlled, but the orders in which it was for the movement of the robot continued in operation until the 23 meters approximate in which I present difficulties. For the tests with the Wifi network was carried out in a property of 95 square meters and 4 floors, where the tour began from the first floor of the property, where it presented difficulty in both movement and video transmission, was on the steps of the third floor to the fourth floor of the property.

\section{RESULTS AND DISCUSSION}

The implemented prototype of the tactical robot can be seen in Fig. 3. The left panel of Fig. 3 shows the electronic parts that was used for the operation of the tactical robot, which balance the weight on the whole surface, since it facilitates to maintain the equilibrium when passing obstacles. On the right panel of Fig. 3 the whole chassis which was made of PVC material is shown. This chassis was joined with PVC cement, so that each part of the joints is attached, which has 4 elbows of $45^{\circ}$ and 6 elbows of $90^{\circ}$ of PVC. The platform which has all the electronic components is fastened with 6 screws of 3 inches by $1 / 4$ on each side. 


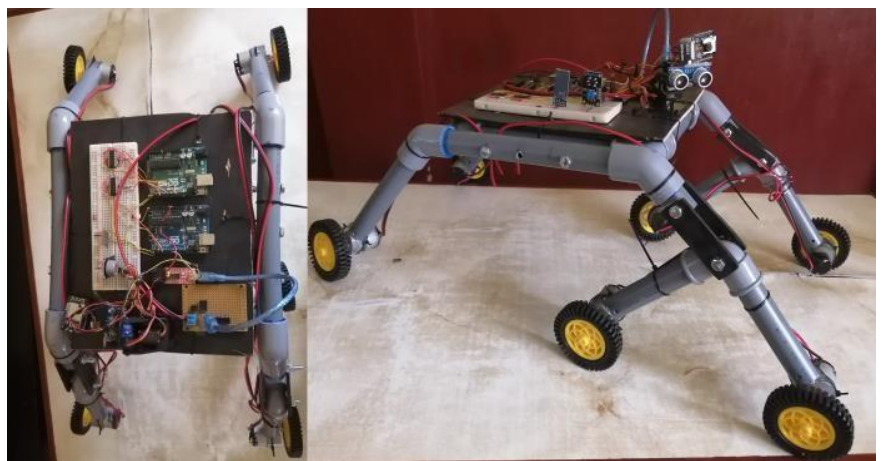

Fig. 3 Left: Assembly of the electronic components. Right: Assembling of the structure of the tactical robot

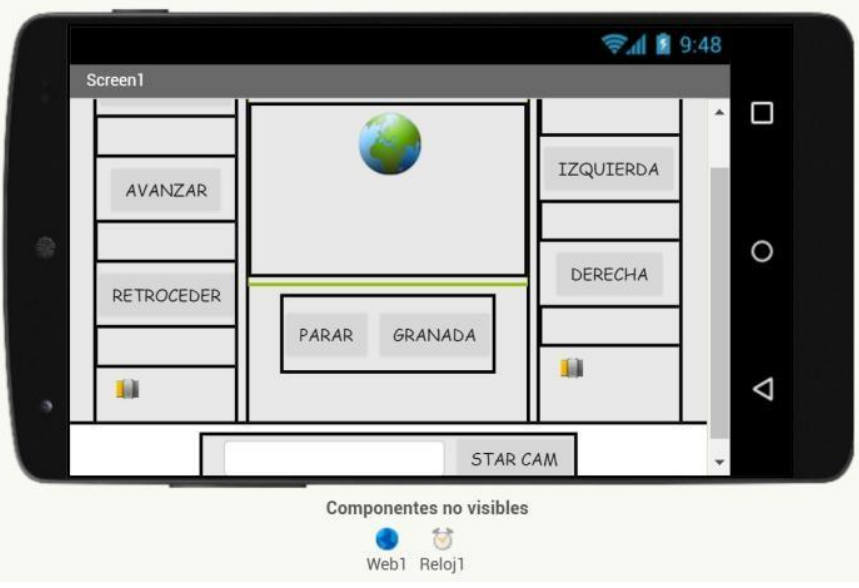

Fig. 4 Tactical Robot Control Interface.

To verify the displacement operation of the tactical robot, the tests were made in the Arduino serial plotter and later with the commands that were made for the control through the telemetry system using the smartphone. Fig. 4 shows the application elaborated for the smartphone with appInventor. On the other hand, it was possible to verify that by means of a good connectivity to the Wi-Fi network the wireless communication will work in an efficient way. Nevertheless, if the connectivity varies the live transmission that is made through the camera will be affected.

Through the different tests over the course of a day, which were in the morning, afternoon and night, it could be observed that the temperature of the environment has an interference through the connectivity of the telemetry system of the tactical robot with the smartphone, since this causes to overheat the device indirectly. Therefore, where there was a greater delay of the communication between the tactical robot and the smartphone, was in the course of the afternoon when the temperature was at an average of $28^{\circ} \mathrm{C}$ (in Lima, the capital city of Peru).

The company FLIER has developed the uPoint multirobot control system which uses the FirstLook microcontroller [11]. This robot includes a rugged touch screen tablet that allows the operator to select from the family of connected robots. Since the controller is based on the well-known Android system, the learning curve for robot operators its significantly reduced. uPoint also features superior wireless communications. The MPU5 radio, which operates in the MANET Wave Relay $\mathbf{1 8}^{\text {th }}$ LACCEI International Multi-Conference for Engineering, Education, and Technology: "Engineering, Integration, and Alliances for a Sustainable Development" "Hemispheric Cooperation for Competitiveness and Prosperity on a Knowledge-Based Economy", 29-31 July 2020, Buenos Aires, Argentina. network, allows the robots to go deeper into complex structures and further towards the target than ever before [11].

Compared to the FirstLook made by FLIER, its control system is made in an Android system as well as the work presented, because its simpler for the users and the learning range is reduced as mentioned above, but nevertheless FirstLook has a connectivity for a massive control of different sibling robots through the same device, which compared to the work presented can only control one independently. Apart from the fact that the system developed by FirstLook is by radio which has the MANET Wave Relay network, as mentioned above, making it more robust in connectivity compared to the tactical robot developed in this work which has a gap of connectivity when exposed to temperatures of $28^{\circ} \mathrm{C}$ on average.

To sum up, there were some difficulties with the transmission of video, this due to the overheating of the system, or even more likely by the environment itself.

At the time of making the calculation for the torque of the motors, equation 1 was taken into account, which facilitated the displacement of the tactical robot. Since it will have the functionality of an all-terrain vehicle, a better torque is required with respect to its total load so that it has a better displacement.

$$
\text { Torque }=\text { Force } \mathrm{x} \text { Distance }(\text { lever arm) }
$$

For the type of tire that was used, two types of experimental tests were made, which compared one type of intermediate tire and another type of blocked tire, which in the experimental test it was concluded that the blocked tire had a better grip on uneven surfaces, thus giving a better grip compared to the intermediate tire.

\section{CONCLUSIONS AND FUTUREWORK}

The conclusive results of the tactical robot have shown that it has considerable efficiency for its first stage. Regarding the live transmission that the tactical robot has, it was fulfilling that the images could be visualized live through the interface created. However, given the factors of the environment and overheating of the device itself, this caused that according to the time of use the live transmission was done in a slower way as mentioned in lines above. Regarding the wireless communication for the control of the tacit robot it had a greater efficiency compared to the live transmission of the video, because in the given experimental tests it was possible to verify that at the moment of giving the order of a direction to the tactical robot, this responded immediately, thus having a good control of the displacement. Nevertheless, given that the tests of this first prototype of tactical robot use a Wi-Fi network it has a reduced range to 20 meters around in closed spaces and 60 meters around in open spaces. In addition, the model of the tires used was of great help given the results of the experimental tests carried out. With the model of the tactical tire, it had a better grip on the ground and on very irregular surfaces its efficiency decreased.

Some improvements for the tactical robot are that it can have a better transmission of video to real time without any delay. Besides it can count as well with a system of nocturnal vision. With regards to the control of the car, this can improve the displacement with better commands which can simplify certain movements and thus in that way making more fluid its 
displacement. The selected motors need to be evaluated due to their efficiency. Because the tires used were narrow, they will be changed to wider ones in case we continue with the present model. However, we are thinking of changing the tactic robot a tank type version to increase its robustness.

For the wireless communication mode, the corresponding studies are being made, in order to have the best response action from transmitter to receiver. Due to the very diverse geographical situations in Peru, the challenge in making this tactical robot design is in the wireless communication mode that it may be counting on, which is expected to find the best communication option.

\section{ACKNOWLEDGMET}

The authors are very thankful to Dr. Carlos SotomayorBeltran for his insightful comments and helpful suggestions in improving this article

\section{REFERENCES}

[1] M. Taher U. Zaman, M. Ahmed, S. H, S. H, A. Jamal, M. Alam, M. Polash, and M. Abdullah A. Amin, "Design and construction of a multipurpose robot," International Journal of Automation, Control and Intelligent Systems, vol. 1, pp. 34-46, 072015.

[2] M. J'ozsef, "Concept of an ugv with arduino device," Hadm'ern"ok Katonai M"uszaki Tudom’anyok Online, vol. IX., pp. 140-148., 012014.

[3] S. Jafri, A. Z. Naqvi, M. Singh, and A. Thorat, "Intelligent tactical robotics," 2016.

[4] N. Andriani, M. Maulida, Y. Aniroh, and M. Alfafa, "Pressure control of a wheeled wall climbing robot using proporsional controller," 012016 , pp. $124-128$.

[5] M. S. Ghute, K. P. Kamble, and M. Korde, "Design of military surveillance robot," in 2018 First International Conference on Secure Cyber Computing and Communication (ICSCCC), Dec 2018, pp. 270272.

[6] ARMY TECHNOLOGY. (2019) Micro tactil ground robot (mtgr). [Online]. Available: https://www.army-technology.com/projects/microtactical-ground-robot-mtgr/

[7] ECA GROUP make sure (2019) Cameleon LG E [Online]. Available: https://www.ecagroup.com/en/solutions/cameleon-lg-e-ugv-unmannedground-vehicle.

[8] SDR TACTICAL ROBOTS (2019) LT/2F "Bulldog" [Online]. Available: http://sdrtactical.com/LT2Arm-Bulldog/

[9] ICOR TCHNOLOGY (2019) EOD Mini-CALIBER ${ }^{\circledR}$ (2019) [Online]. Available: https://icortechnology.com/robots/mini-caliber/

[10]ZONA TACTICA (2018) NATIONAL TACTICAL OFFICERS ASSOCIATION [Online]. Available: https://www.flir.es/products/firstlook/

[11]FLIR. (2019) Flir firstlook. [Online]. Available: https://www.flir.es/products/firstlook/ 